\title{
Antimicrobial effect of alexidine and chlorhexidine against Enterococcus faecalis infection
}

\author{
Hyun-Shik Kim ${ }^{1, *}$, Seok Woo Chang ${ }^{2, *}$, Seung-Ho Baek ${ }^{1}$, Seung Hyun Han ${ }^{3}$, Yoon Lee ${ }^{4}$, Qiang Zhu ${ }^{5}$ \\ and Kee-Yeon Kum ${ }^{1}$
}

A previous study demonstrated that alexidine has greater affinity for the major virulence factors of bacteria than chlorhexidine. The aim of this study was to compare the antimicrobial activity of $1 \%$ alexidine with that of $2 \%$ chlorhexidine using Enterococcus

faecalis-infected dentin blocks. Sixty bovine dentin blocks were prepared and randomly divided into six groups of 10 each. E. faecalis was inoculated on 60 dentin blocks using the Luppens apparatus for $24 \mathrm{~h}$ and then the dentin blocks were soaked in $2 \%$ chlorhexidine or $1 \%$ alexidine solutions for 5 and $10 \mathrm{~min}$, respectively. Sterile saline was used as a control. The antimicrobial efficacy was assessed by counting the number of bacteria adhering to the dentin surface and observing the degradation of bacterial shape or membrane rupture under a scanning electron microscope. Significantly fewer bacteria were observed in the $2 \%$ chlorhexidine- or $1 \%$ alexidine-soaked groups than in the control group $(P<0.05)$. However, there was no significant difference in the number of bacteria adhering to the dentinal surface between the two experimental groups or between the two soaking time groups $(P>0.05)$. Ruptured or antiseptic-attached bacteria were more frequently observed in the 10-min-soaked chlorhexidine and alexidine groups than in the 5 -min-soaked chlorhexidine and alexidine groups. In conclusion, 10 -min soaking with $1 \%$ alexidine or $2 \%$ chlorhexidine can be effective against $E$. faecalis infection.

International Journal of Oral Science (2013) 5, 26-31; doi:10.1038/ijos.2013.11; published online 15 March 2013

Keywords: alexidine; antibacterial effect; chlorhexidine; Enterococcus faecalis; Luppens apparatus; root canal soaking; scanning electron microscope

\section{INTRODUCTION}

Elimination of microbial contamination from the root canal system at the time of canal obturation is a prerequisite for successful outcomes in endodontic therapy. ${ }^{1}$ Bacteria in the root canal are present either as free-floating single cells or attached to each other or to the root canal walls to form a biofilm..$^{2-3}$ Enterococcus faecalis, a Gram-positive and facultative anaerobic bacterium, is more likely to be found in persistent infections than in primary infections. ${ }^{4}$ The inherent ability of $E$. faecalis to adhere and invade dentinal tubules ${ }^{5-6}$ and form communities in an organized biofilm may contribute to bacterial resistance and persistence of infection after root canal treatment. ${ }^{7}$ Thus, many studies have been conducted on E. faecalis-infected dentin blocks to determine the antimicrobial activity of intracanal disinfectants. ${ }^{8-9}$ Mechanical instrumentation alone does not result in total or permanent reduction of bacteria from infected root canals ${ }^{10}$ and thus, requires the concomitant use of various antimicrobial irrigants in a sequential manner or in combination to enhance the antimicrobial effect. ${ }^{11}$
Chlorhexidine (CHX) digluconate is a bisguanide disinfectant that has antimicrobial substantive activity, ${ }^{12-14}$ and thus has been widely used as an auxiliary canal irrigant or a canal soaking agent against $E$. faecalis ${ }^{15-16} \mathrm{~A}$ recent study also demonstrated that $\mathrm{CHX}$ attenuates the activity of E. faecalis lipoteichoic acid (LTA). ${ }^{17}$ However, CHX was shown to have no tissue solvent activity. ${ }^{15}$

Alexidine (ALX) is also a bisguanide disinfectant that has greater affinity for the major virulence factors such as bacterial lipopolysaccharide and LTA than CHX. ${ }^{18}$ It was also reported that the interaction of ALX and sodium hypochlorite did not form an insoluble precipitate known as para-chloroaniline. ${ }^{19}$ However, to date, its antimicrobial efficacy on E. faecalis biofilms is unknown. Therefore, the aim of this study was to compare the antimicrobial activity of $1 \%$ ALX with that of $2 \%$ CHX using E. faecalis-infected bovine dentin blocks. The hypothesis was that $1 \%$ ALX and $2 \%$ CHX would show no difference in antibacterial efficacy against $E$. faecalis.

\footnotetext{
${ }^{1}$ Department of Conservative Dentistry, Dental Research Institute and BK21 Program, Seoul National University Dental Hospital, Seoul National University School of Dentistry, Seoul, Korea; ${ }^{2}$ Department of Conservative Dentistry, School of Dentistry, Kyung Hee University, Seoul, Korea; ${ }^{3}$ Department of Oral Microbiology and Immunology, Dental Research Institute and BK21 Program, Seoul National University Dental Hospital, Seoul National University School of Dentistry, Seoul, Korea; ${ }^{4}$ Department of Dentistry Wonju College of Medicine, Yonsei University, Wonju, Korea and ${ }^{5}$ Division of Endodontology, School of Dental Medicine, University of Connecticut Health Center, Farmington, USA

*These authors contributed equally to this work.

Correspondence: Dr KY Kum, 25-9 Jongro-Gu Yungun-Dong, Department of Conservative Dentistry, Dental Research Institute and BK21 Program, Seoul National University Dental Hospital, Seoul National University School of Dentistry, Seoul 110-768, Korea
}

E-mail: kum6139@snu.ac.kr

Received 25 October 2012; accepted 27 January 2013 


\section{MATERIALS AND METHODS}

\section{Preparation of dentin blocks}

Bovine incisor teeth were extracted, and the roots were cleaned with scalers and $4 \%$ sodium hypochlorite $(\mathrm{NaOCl})$ solution. The coronal and apical thirds of the roots were removed using a Microtome (Struers, Rodovre, Denmark). The middle thirds of the roots were split along the long axis. The specimens were immersed in $4 \%$ $\mathrm{NaOCl}$ for $5 \mathrm{~min}$ and then $17 \%$ ethylene diaminetetraacetic acid (EDTA) for 2 min to remove the smear layer, then washed with distilled water and sterilized by autoclaving.

\section{E. faecalis inoculation}

E. faecalis (ATCC 29212) inoculation was carried out using the apparatus reported by Luppens et al. ${ }^{20}$ The apparatus consisted of a vessel containing 10-fold-diluted brain-heart infusion (BHI; Difco Laboratories, Detroit, MI, USA), a pump (Masterflex pump system; Cole-Parmer Instrument Co., Chicago, IL, USA), a culture container (perfusion culture container 1301; Minucells and Minutissues, Bad Abbach, Germany), and a vessel for waste, all of which were connected with silicone tubes (Figure 1). Before inoculation, samples were placed in the culture container, and BHI was pumped through the system for $30 \mathrm{~min}$. To inoculate $E$. faecalis, culture media (BHI) in the container was removed. The 24 -h culture $(15 \mathrm{~mL})$ of E. faecalis $\left(5.5 \times 10^{9}\right.$ colonyforming units per $\mathrm{mL}$ ) was then added to the container. After $30 \mathrm{~min}$, the pump was restarted, and samples were allowed to develop a biofilm for $24 \mathrm{~h}$ in the presence of a constant nutrient flow. After the pump was stopped, the blocks were removed from the container and placed into the cell culture wells ( 1 dentin block per well) of a 24 -well plate.

\section{Preparation of antibacterial solutions}

The dentin blocks were soaked in $1 \mathrm{~mL}$ of $2 \%$ CHX or $1 \%$ ALX for 5 and $10 \mathrm{~min}$, respectively. A $2 \%$ solution of $\mathrm{CHX}$ was prepared by diluting a 20\% solution of CHX (C 9394; Sigma-Aldrich, St Louis,
MO, USA) with sterile distilled water. A $1 \%$ solution of ALX was prepared by dissolving ALX dihydrochloride powder (M68182628; Gentaur, Kampenhout, Belgium) in sterile distilled water. We tested 1\% concentration of ALX solution because ALX with a concentration higher than $1 \%$ caused moderate cytotoxicity against human gingival fibroblasts in our preliminary study. Sterile saline was used as the control group.

Scanning electron microscopic observation and statistical analysis After the aforementioned treatment, all dentin blocks were fixed in $2 \%$ glutaraldehyde at $4{ }^{\circ} \mathrm{C}$ and prepared for observation under a scanning electron microscope (SEM). The central beam of the SEM (Hitachi S-4700, Tokyo, Japan) was directed to the surface of each dentin block under 50 times magnification in order to observe the whole sample surface. Then, 10 areas of each specimen projected onto the screen were randomly selected and magnified 1500 times. The number of bacteria observed within the grid was counted. To observe the shape of bacteria and their characteristics, areas of interest were photographed under 10000 and 20000 times magnification. The final results for the mean number of $E$. faecalis binding to dentin were obtained by calculating the mean scores of the 10 selected areas of each specimen. ${ }^{21}$ The data were analyzed using one-way ANOVA, and the comparison of means was conducted using the Tukey post hoc multiple comparison test. $P$ value less than 0.05 was considered statistically significant.

\section{RESULTS}

The numbers of bacteria counted in three different groups are summarized in Table 1 . In the groups soaked with $2 \%$ CHX or $1 \%$ ALX for 5 min, significantly fewer bacterial cells were observed than in the saline (control) group $(P=0.002)$, but there was no significant difference in the number of bacteria adhering to the dentinal surface between the two experimental groups $(P=0.869)$. In the groups soaked with $2 \%$ CHX or $1 \%$ ALX for $10 \mathrm{~min}$, the number of bacteria

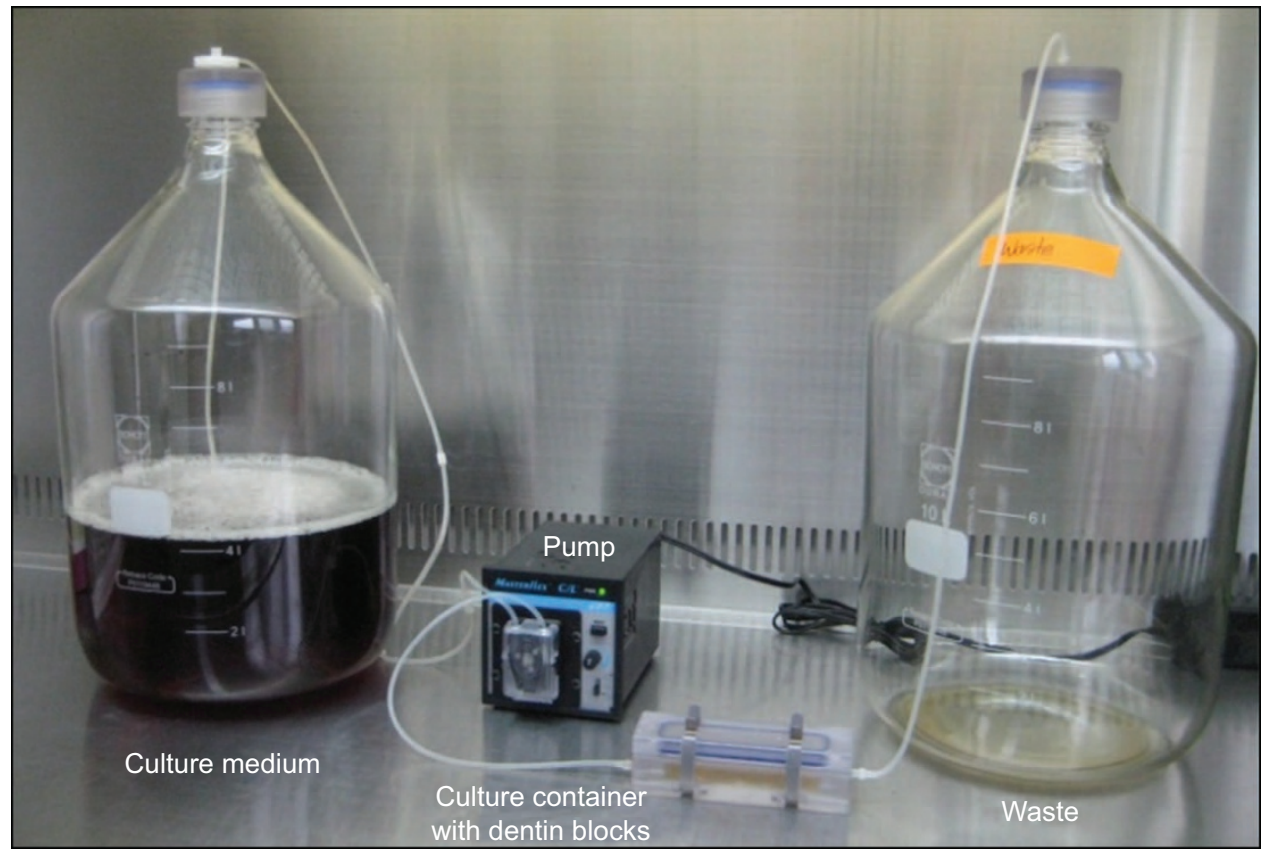

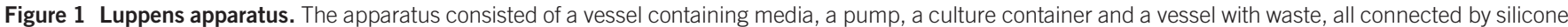
tubing. 
Table 1 Cell numbers of Enterococcus faecalis adhered to bovine dentin in each treatment group

\begin{tabular}{|c|c|c|c|c|}
\hline Soaking time/min & Irrigant solution & Sample number & No. of cells/observation spot ${ }^{a}$ & Significant difference \\
\hline \multirow[t]{3}{*}{5} & Saline & 10 & $90 \pm 12.86$ & \\
\hline & $2 \% \mathrm{CHX}$ & 10 & $18 \pm 7.37$ & $\mathrm{~b}, \mathrm{c}$ \\
\hline & $1 \% \operatorname{ALX}$ & 10 & $16 \pm 4.68$ & $\mathrm{~b}, \mathrm{c}$ \\
\hline & $2 \% \mathrm{CHX}$ & 10 & $16 \pm 3.67$ & $b, c$ \\
\hline & $1 \% \operatorname{ALX}$ & 10 & $14 \pm 4.27$ & $\mathrm{~b}, \mathrm{c}$ \\
\hline
\end{tabular}

ALX, alexidine; $\mathrm{CHX}$, chlorhexidine.

${ }^{a}$ Mean \pm s.d.

${ }^{\text {b }}$ Significant difference at $P<0.05$ compared with saline (control) group.

${ }^{c}$ Insignificant difference at $P>0.05$ compared with $2 \% \mathrm{CHX}$ or $1 \% \mathrm{ALX}$ group.

was significantly reduced compared with the control group $(P=$ $0.004)$. However, there was no statistically significant difference in the number of adhered bacteria between the ALX and CHX groups $(P=0.674)$.
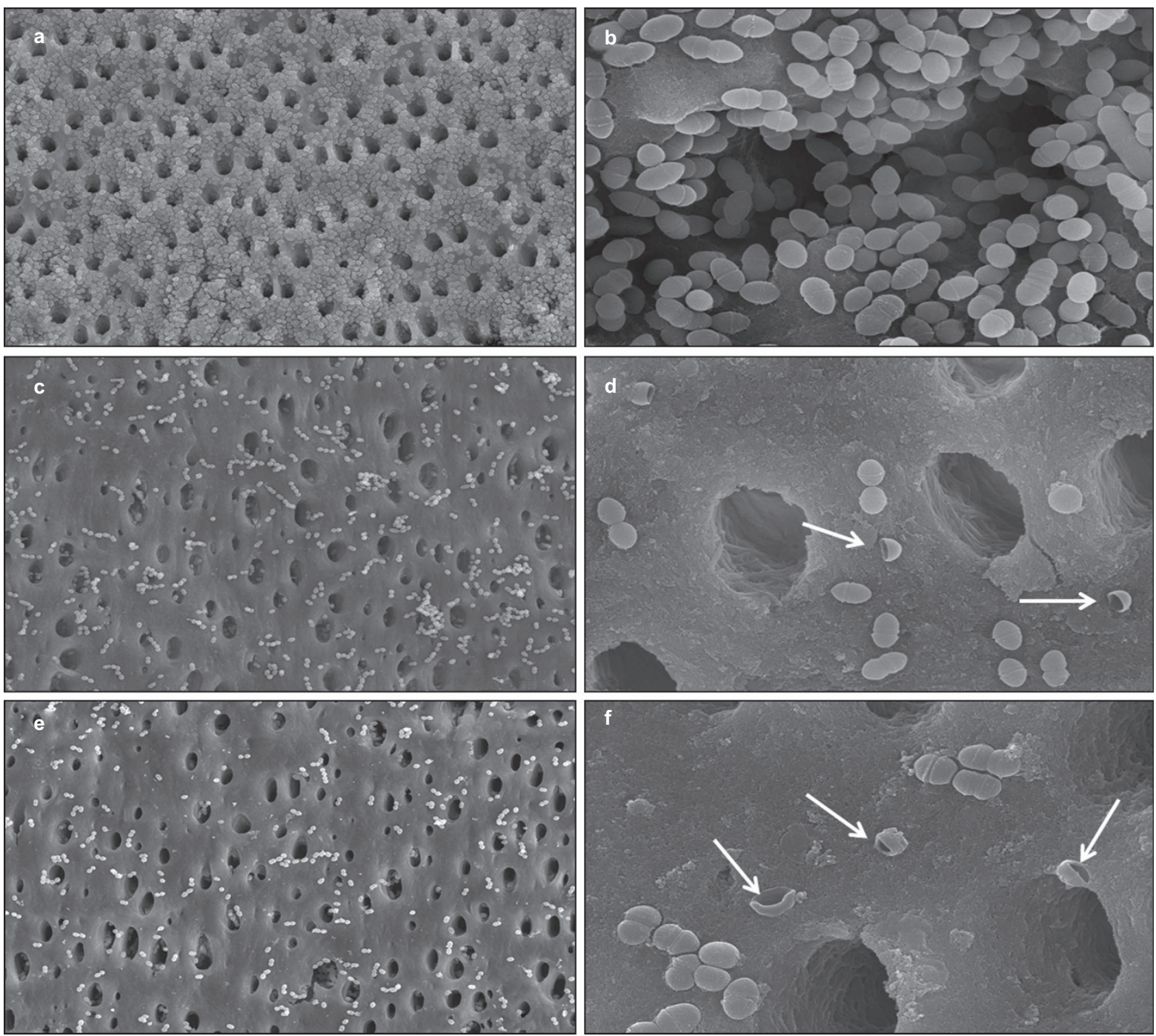

Figure $2 \mathrm{SEM}$ images of $\boldsymbol{E}$. faecalis-infected dentin blocks in the 5-min-soaked groups. SEM images of $E$. faecalis-infected dentin blocks treated with saline show a large number of adhering bacteria $(\mathbf{a}, \times 1500)$ with intact bacterial membranes $(\mathbf{b}, \times 20000)$. The group treated with $2 \% \mathrm{CHX}$ shows reduced numbers of adhering bacteria $(\mathbf{c}, \times 1500)$ and many lysed $E$. faecalis $(\mathbf{d}, \times 10000$, white arrow). The group treated with ALX also shows fewer adhering bacteria $(\mathbf{e}, \times 1500)$ with damaged membranes (f, $\times 10000$, white arrow). ALX, alexidine; CHX, chlorhexidine; E. faecalis, Enterococcus faecalis; SEM, scanning electron microscope. 

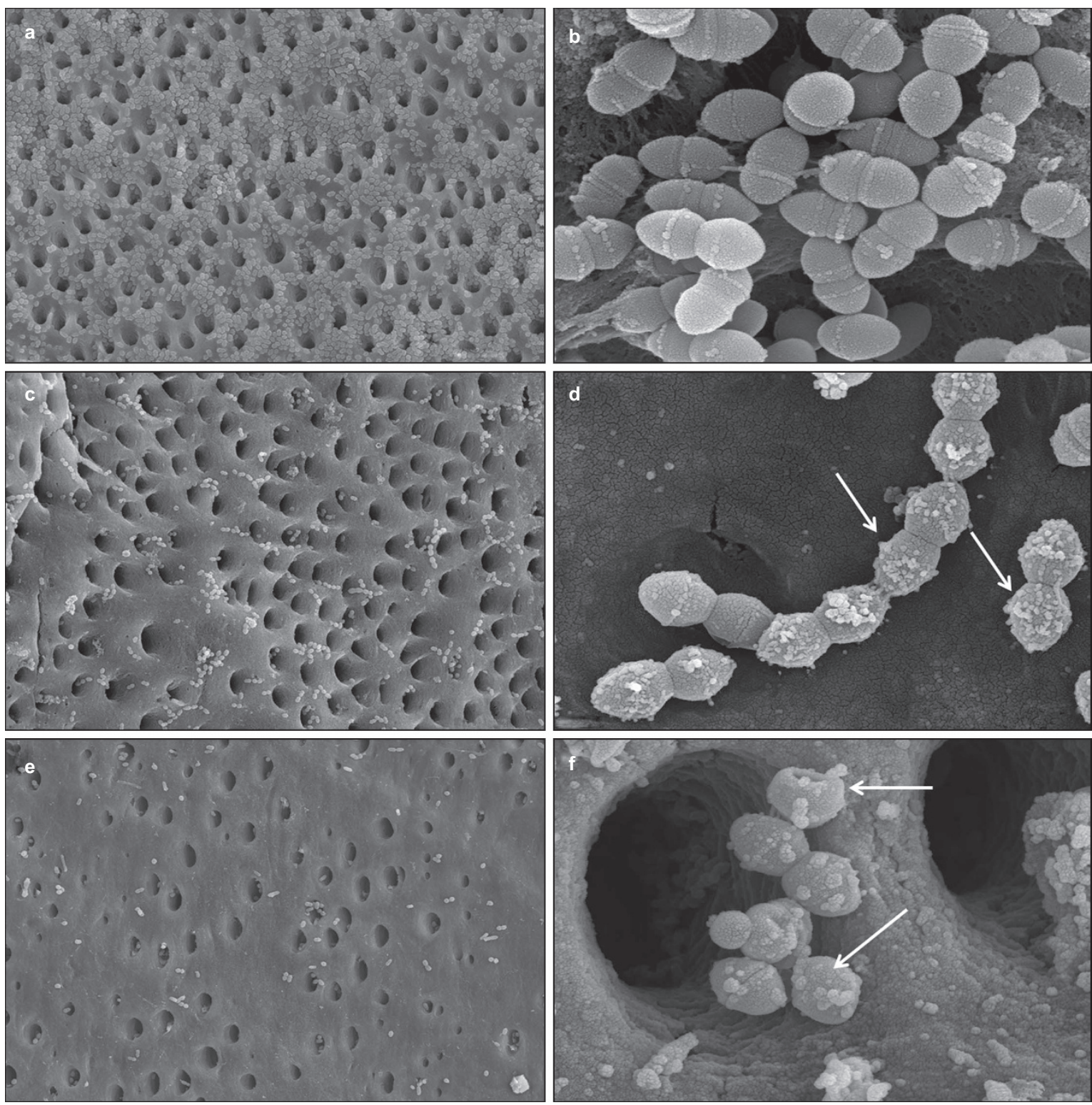

Figure 3 SEM images of $\boldsymbol{E}$. faecalis-infected dentin blocks in the 10-min-soaked groups. SEM images of $E$. faecalis-infected dentin blocks treated with saline for 10 min show many adhering $E$. faecalis $(\mathbf{a}, \times 1500)$ with normal shape $(\mathbf{b}, \times 20000)$. The group soaked with $2 \% \mathrm{CHX}$ shows fewer adhering bacteria $(\mathbf{c}, \times 1500)$ and $\mathrm{CHX}$ particles attached to bacterial membranes (d, $\times 20000$, white arrow). The group treated with $1 \%$ ALXalso shows fewer adhering bacteria with abnormal shape (e, $\times 1500$ ) and ALX particles attached to bacterial membranes (f, $\times 20000$, white arrow). ALX, alexidine; CHX, chlorhexidine; E. faecalis, Enterococcus faecalis; SEM, scanning electron microscope.

CHX groups (Figure 2c-2f). No remarkable difference in bacterial shape could be seen between control groups soaked with saline for 5 or $10 \mathrm{~min}$ (Figures 2a, 2b, 3a and 3b). However, antiseptic particles were more frequently attached to the bacterial surface in the 10-minsoaked CHX and ALX groups (Figure 3c-3f) than the 5-min-soaked $\mathrm{CHX}$ and ALX groups (Figure 2c-2f). In particular, damaged or antiseptic-attached bacteria were frequently observed in the 10 -minsoaked ALX group (Figure 3f).

\section{DISCUSSION}

This study supports the hypothesis that $1 \%$ ALX and 2\% CHX have no difference in antibacterial activity against E. faecalis. In the present study, 5-min soaking with $2 \%$ CHX or $1 \%$ ALX significantly reduced the number of bacteria adhering to the dentinal surface compared to the control group, but the antiseptic-attached bacteria were scarcely observed. On the contrary, in the 10-min soaking with CHX or ALX groups, antiseptic particles attached to the bacterial surface and abnormal bacterial shapes were commonly found, particularly in the ALX group. These findings imply two clinically important insights. One is that the antibacterial activity of ALX or CHX is due to the adherence of antiseptic particles to the bacterial cell wall. The other is that maximum adherence of antiseptic particles to the bacterial cell wall took longer than $5 \mathrm{~min}$. The clinical implication of these findings is that ALX or CHX should be in direct contact with the infected dentinal surface for a prolonged time ( $>5 \mathrm{~min}$ ) in order to achieve their maximum antibacterial effect against E. faecalis. In general, Gram-positive 
bacteria like E. faecalis are more sensitive to cations, because they are negatively charged. Chlorhexidine is a cationic bisguanide that seems to act by adhering onto the negatively charged phosphate groups of bacterial cell walls, causing leakage of intracellular components. ${ }^{22}$ At higher concentrations, as were used in this study, CHX has a bactericidal effect due to precipitation and/or coagulation of the cytoplasm, probably caused by protein cross-linking. ${ }^{23}$ ALX, similar to $\mathrm{CHX}$, is a cationic bisguanide that induces lipid phase separation and domain formation at bacterial membranes. ${ }^{24}$ However, ALX has greater affinity for bacterial LTA than CHX. ${ }^{19}$ This characteristic might result in the formation of many ruptured (damaged) or antiseptic-attached bacteria in the 10-min-soaked ALX group. Furthermore, both irrigants have substantive antimicrobial activity. ${ }^{25}$ This can allow ALX to be applied as a supplementary final rinse before intracanal medication or a canal soaking agent before canal obturation, as well as a drug component of controlled release device. ${ }^{26}$

The inoculation system used in this study was the method utilized by Luppens et al. ${ }^{20}$ The Luppens apparatus has several advantages over previous methods suggested by Das et al. ${ }^{27}$ and Wright et al. ${ }^{28}$ First, the Luppens method counts bacterial cell number rather than determining the minimum inhibitory concentration of the antibacterial agent, which is less appropriate since growth-inhibited cells (e.g., E. faecalis) can still recover, regrow and reinfect the root canal system. Second, the Luppens method applies shear force and poor nutritional supply when creating the biofilm. The resulting biofilms with firmly attached bacteria more closely simulate the biofilms formed in the infected root canal system. Finally, the Luppens apparatus is simple and economical compared to previous methods such as the Robbins device. ${ }^{29}$

A recent study emphasized the importance of standardization of factors such as biofilm age when comparing the effectiveness of disinfecting agents against biofilm bacteria. ${ }^{30}$ Regarding this issue, a previous study assessed the efficacy of $\mathrm{CHX}$ and cetrimide in eradicating 1-day-old biofilms of E. faecalis. ${ }^{31}$ Another study reported that 1-dayold biofilms of $E$. faecalis provide a better comparison of the antimicrobial effectiveness of disinfectants than 3-day-old biofilms. ${ }^{32}$ The study revealed that 3-day-old biofilms showed a natural decrease in adhered bacteria with additional 1-day incubation despite the absence of an antibacterial agent. On the contrary, 1-day-old biofilms showed no change in the number of adhered bacteria with additional 1-day incubation. This implies that 3-day-old biofilms could have 'natural' loss of adhered bacteria not caused by an antibacterial irrigant. Considering this report, we used the Luppens apparatus to yield 1day-old biofilms of $E$. faecalis, which might be a sufficient time period for obtaining adequate biofilm density. ${ }^{32-33}$

In the present study, the SEM was only used to observe the numbers or the degradation of bacterial shape or membrane rupture of bacteria attached on dentin substrate, but not their viability. Therefore, alternative viability evaluation such as LIVE/DEAD bacterial viability test with a confocal laser scanning microscope would be helpful in evaluation of the antimicrobial efficacy, in future study. Also, the present study did not include sodium hypochlorite as a positive control because its antimicrobial mode against $E$. faecalis is different from that of ALX and CHX. Sodium hypochlorite kills E. faecalis by the high alkaline $\mathrm{pH}^{34}$ but the ALX and CHX attach to the bacterial membrane surface, cause leakage of intracellular components and rupture bacterial membranes, which are clearly shown in the SEM observation of our study.

Although the present study showed that $1 \%$ ALX was effective against $E$. faecalis infection, we recommend ALX as a supplementary final rinse before intracanal medication or as canal soaking agent before canal obturation in persistently-infected/failed root canals, because ALX does not possess organic tissue-dissolving properties like sodium hypochlorite. ${ }^{35}$ In addition, ALX was studied to be a possible replacement of $\mathrm{CHX}$ in root canal irrigation, since the interaction of ALX and sodium hypochlorite did not form an insoluble precipitate known as para-chloroaniline. ${ }^{19}$ Therefore, strategies to treat E. faecalis-infected root canals may benefit from mechanical disruption of the multicellular bacterial structure by nickel-titanium instrumentation, dissolution of the extracellular polymeric substance by $\mathrm{NaOCl}$ irrigation, supplementary rinse with ALX before intracanal medication, calcium hydroxide medication, and canal soaking with ALX or CHX solution for $10 \mathrm{~min}$ before canal obturation. This treatment protocol may synergistically improve the success rate of endodontic treatment in E. faecalis-infected root canals.

\section{CONCLUSION}

Under the limitation of the present study, 1\% ALX has a similar antibacterial effect to 2\% CHX against E. faecalis, suggesting that both irrigants can be useful for a supplementary final rinse before intracanal medication or as canal soaking agents before canal obturation in E.faecalis-infected root canals.

\section{ACKNOWLEDGEMENTS}

This study was supported by the National Research Foundation (NRF) of Korea funded by the Ministry of Education, Science and Technology (MEST) (No.2009-0086835, 2011-0014231, 2012-0008693: Drs KY Kum, SH Han and SW Chang), South Korea.

1 Sjögren U, Figdor D, Persson S et al. Influence of infection at the time of root filling on the outcome of endodontic treatment of teeth with apical periodontitis. Int Endod J 1997; 30(5): 297-306.

2 Rucucci D, Siqueira JF Jr. Biofilms and apical periodontitis: study of prevalence and association with clinical and histopathologic findings. J Endod 2010; 36(8): 1277-1288.

3 Haapasalo M, Endal U, Zandi $\mathrm{H}$ et al. Eradication of endodontic infection by instrumentation and irrigation solution. Endod Topics 2005; 10(1): 77-102.

4 Siren EK, Haapasalo MPP, Ranta K et al. Microbial findings and clinical treatment procedures in endodontic cases selected for microbiological investigation. Int Endod $J$ 1997; 30(2): 90-95.

5 Sedgley CM, Lennan SL, Appelbe OK. Survival of Enterococcus faecalis in root canals ex vivo. Int Endod J 2005; 38(10): 735-742.

6 Chivatxaranukul P, Dashper SG, Messer HH. Dentinal tubule invasion and adherence by Enterococcus faecalis. Int Endod J 2008; 41(10): 873-882.

7 Distel JW, Hatton JF, Gillespie MJ. Biofilm formation in medicated root canals. J Endod 2002; 28(10): 689-693.

8 Giardino L, Ambu E, Savoldi E et al. Comparative evaluation of antimicrobial efficacy of sodium hypochlorite, MTAD, and Tetraclan against Enterococcus faecalis biofilm. $J$ Endod 2007; 33(7): 852-855.

9 Arias-Moliz MT, Ferrer-Luque CM, Espigares-Garcia M et al. Enterococcus faecalis biofilms eradication by root canal irrigants. J Endod 2009; 35(5): 711-714.

10 Byström A, Sundqvist G. Bacteriologic evaluation of the efficacy of mechanical root cana instrumentation in endodontic therapy. Scand J Dent Res 1981; 89(4): 321-328.

11 Zehnder M. Root canal irrigants. J Endod 2006; 32(5): 389-398.

12 Parsons GJ, Patterson SS, Miller CH et al. Uptake and release of chlorhexidine by bovine pulp and dentin specimens and their subsequent acquisition of antibacterial properties. Oral Surg Oral Med Oral Pathol Oral Radiol 1980; 49(5): 455-459.

13 Basrani B, Santos JM, Tjaderhane L et al. Substantive antimicrobial activity in chlorhexidine-treated human root dentin. Oral Surg Oral Med Oral Pathol Oral Radiol Endod 2002; 94(2): 240-245.

14 Baca P, Junco P, Arias-Moliz MT et al. Antimicrobial substantivity over time of chlorhexidine and cetrimide. J Endod 2012; 38(7): 927-930.

15 Mohammadi Z, Abbott PV. The properties and applications of chlorhexidine in endodontics. Int Endod J 2009; 42(4): 288-302.

16 Gomez BP, Ferraz CC, Vianna ME et al. In vitro antimicrobial activity of several concentrations of sodium hypochlorite and chlorhexidine gluconate in the elimination of Enterococcus faecalis. Int Endod J 2001; 34(6): 424-428.

17 Lee JK, Baik JE, Yun $\mathrm{CH}$ et al. Chlorhexidine gluconate attenuates the ability of lipoteichoic acid from Enterococcus faecalis to stimulate toll-like receptor 2. J Endod 2009; 35(2): 212-215.

18 Zorko M, Jerala R. Alexidine and chlorhexidine bind to lipopolysaccharide and lipoteichoic acid and prevent cell activation by antibiotics. J Antimicrob Chemoth 2008; 62(4): 730-737. 
19 Kim HS, Zhu Q, Han SH et al. Chemical interaction of alexidine and sodium hypochlorite. J Endod 2012; 38(1): 112-116.

20 Luppens SB, Reij MW, van der Heijden RW et al. Development of a standard test to assess the resistance of Staphylococcus aureus biofilm cells to disinfectants. App Environ Microbiol 2002; 68(9): 4194-4200.

21 Yang SE, Cha JH, Kim ES et al. Effect of smear layer and chlorhexidine treatment on the adhesion of Enterococcus faecalis to bovine dentin. J Endod 2006; 32(7): 663-667.

22 Greenstein G, Berman C, Jaffin R. Chlorhexidine. An adjunct to periodontal therapy. J Periodontol 1986; 57(6): 370-376.

23 Kontakiotis $\mathrm{E}$, Nakou M, Georgopoulou M. In vitro study of the indirect action of calcium hydroxide on the anaerobic flora of the root canal. Int Endod J 1995; 28(6): 285-289.

24 Baker PJ, Coburn RA, Genco RJ et al. Structural determinants of activity of chlorhexidine and alkyl bisbiguanides against the human oral flora. J Dent Res 1987; 66(6): 1099-1106.

25 Roberts WR, Addy M. Comparison of the bisbiguanide antiseptics alexidine and chlorhexidine: I. Effect on plaque accumulation and salivary bacteria. J Clin Periodontol 1981; 8(3): 213-219.

26 Lee $\mathrm{Y}$, Han SH, Hong SH et al. Antimicrobial efficacy of a polymeric chlorhexidine release device using in vitro model of Enterococcus faecalis infected dentinal tubule infection. J Endod 2008; 34(7): 855-858.

27 Das JR, Bhakoo M, Jones MV et al. Changes in the biocide susceptibility of Staphylococcus epidermidis and Escherichia coli cells associated with rapid attachment to plastic surfaces. J Appl Microbiol 1998; 84(5): 852-858.
28 Wright TL, Ellen RP, Lacroix JM et al. Effects of metronidazole on Porphyromonas gingivalis biofilms. J Periodontal Res 1997; 32(5): 473-477.

29 Adams JL, McLean RJ. Impact of rpoS deletion on Escherichia coli biofilms. Appl Environ Microbiol 1999; 65(9): 4285-4287.

30 Shen Y, Stojicic S, Haapasalo M. Antimicrobial efficacy of chlorhexidine against bacteria in biofilms at different stages of development. J Endod 2011; 37(5): 657661.

31 Arias-Moliz M, Ferrer-Luque C, Gonzalez-Rodriquez M et al. Eradiation of Enteroccus faecalis biofilms by cetrimide and chlorhexidine. J Endod 2010; 36(1): 87-90.

32 Lima K, Fava L, Siqueira J. Susceptibilities of Enterococcus faecalis biofilms to some antimicrobial medications. J Endod 2001; 27(10): 616-619.

33 Persoon IF, Hoogenkamp MA, Bury A et al. Effect of vanadium chloroperoxidase on Enterococcus faecalis biofilms. J Endod 2012; 38(1): 72-74.

34 Portenier I, Waltimo TMT, Haapasalo M. Enterococcus faecalis-the root canal survivor and 'star' in post-treatment disease. Endod Topics 2003; 6(1): 135-169.

35 Naenni N, Thoma K, Zehnder M. Soft tissue dissolution capacity of currently used and potential endodontic irrigants. J Endod 2004; 30(11): 785-787.

This work is licensed under a Creative Commons

Attribution-NonCommercial-NoDerivative Works 3.0 Unported License. To view a copy of this license, visit http:// creativecommons.org/licenses/by-nc-nd/3.0 